\title{
Astrometric positions of stars with high proper motions in the Southern Hemisphere ${ }^{\star}$
}

\author{
J.M. Rousseau and J.P. Périé \\ Observatoire de Bordeaux, INSU/CNRS-Université Bordeaux 1, BP. 89, F-33270 Floirac, France
}

Received August 27; accepted December 5, 1996

\begin{abstract}
Several stars with large proper motions, cited by W.J. Luyten, were included in the preliminary programme for the HIPPARCOS mission. When performing preparatory measurements of plates, difficulties were encountered in identifying certain of these stars when relying only on published coordinates. We have taken advantage of this work which relates to the southern sky in order to determine the astrometric position of the greatest possible number of these objects, even for those which were not included in the programme.
\end{abstract}

Key words: astrometry — stars: kinematics

\section{Introduction}

This work is identical in all respects with that described in this journal in relation to the catalogue of the "Stars of the southern hemisphere proposed for the Hipparcos mission" (Rousseau et al. 1996) [RPG 1996] and in the article relating to the "Astrometric positions of stars associated with nebulosities in the southern hemisphere" (Rousseau \& Périé 1996) [RP 1996]. The origin of the work, observations, measuring equipment and procedures, reduction methods, the calculation of the J2000 positions, the calculation of the precision and the epoch are the same since all the objects were processed simultaneously. Out of all the stars in the programme, and included in the list drawn up by W.J. Luyten (1979) and identified by the acronym LHS, we have eliminated:

- Stars which were too bright, and thus did not allow accurate measurements to be made.

- Stars whose identification was uncertain, in spite of the use of the charts published by W.J. Luyten.

* Catalogue is only available in electronic form at the CDS via anonymous ftp to cdsarc.u-strasbg.fr (130.79.128.5) or via http://cdsweb.u-strasbg.fr/Abstract.html
- Stars which had an FK4 identifier. These are astrometric references points whose position is already very well known.

Our list only includes stars with a declination of less than -17.5 degrees. It is not exhaustive. The precision of the coordinates is: $0.25^{\prime \prime}$ in right ascension and $0.29^{\prime \prime}$ in declination

\section{Catalogue description}

The description follows the same order as the catalogue columns.

\section{1. $L H S$}

This is the number given by W.J. Luyten (1979). A number followed by an A or B indicates that this star is included in the ADS or IDS catalogues with this suffix.

\section{2. $M a g$}

These values should only be used as an indication. Various sources have been used:

- The Centre for Astronomic Data at Strasbourg.

- The Hipparcos Input Catalogue.

- LHS CATALOGUE 1979 for faint stars.

- Bessel's list (1990). In this case, the magnitude is preceded by a "b".

- Rodgers \& Eggen's list (1974). In this case, the magnitude is preceded by an "r".

\subsection{J2000 position}

The definition is the same as that already given [RPG 1996] and [RP 1996]. When several observations are available, we have checked, before calculating the mean of the positions, that the continuation of the individual positions is consistent with the value already known of the proper motion. 
2.4. Proper motions, precision, epoch, number of observations

- $\mu_{\alpha} \cos \delta$ : Proper motion in right ascension (in arcseconds per year)

- $\mu_{\delta}$ : Proper motion in declination (in arcseconds per year)

Various sources were used:

- Positions and Proper Motions (Röser \& Bastian 1992).

- Hipparcos Input Catalogue.

- Luyten Half Second Catalogue.

- $\sigma_{\alpha}$ : Root-mean-square error in right ascension, in arcseconds.

- $\sigma_{\delta}$ : Root-mean-square error in declination, in arcseconds

as already defined [RPG 1996] and [RP 1996].

- Ep: Date, to the nearest 0.01 years, of the plate, or the mean date of the observations, if the position is the average of the positions taken from several plates. - $N$ : Number of plates used on which this star is shown. - *: If $N$ is followed by "**, see the notes.

\subsection{Equivalencies}

Several identifiers are given, selected in order of preference from: HD, HIC, PPM, GJ, L, LP, LTT, LFT, BD, CD, $\mathrm{CPD}, \mathrm{V}^{*}$, GJ and $\mathrm{Ci}$. These identifiers, in addition to the acronyms ADS and IDS cited in 2.1, have already been individually described [RPG 1996].

\section{Conclusion}

This work is a supplement to our preparation catalogue for the Hipparcos Mission, and has been compiled in the same way. The catalogue's principal characteristic is its homogeneity, since it has been compiled from plates taken by one instrument, measured using one machine, by just two operators, reduced by one method, and with the results being submitted to identical criteria. Its aim is to define with precision the position of certain rapid stars at a known time. It also enables the proper motion of faint objects to be detected, so long as the proper motion is known, more accurately than from using the positions available up until now.

Acknowledgements. We would like to thank: the Paris-Meudon Observatory for letting us use copies of the plates taken by the ESO Blue Survey, and Mrs C. Turon, who has given us valuable advice and encouragement. This research work has made use of the Simbad database, operated at CDS, Strasbourg, France.

\section{LHS}

10

29

49

$110 / 111$

118

$171 / 172$

222

$289 / 290$

$308 / 309$

$386 / 387$

$415 / 416$

$437 / 438$

442

$442 / 443$

497

520

$1077 / 1078$

$1097 / 1098$

1110

1208

$1337 / 1338$

1409

$1466 / 1467$

1515

$1551 / 1552$

$1724 / 1727$

$1770 / 1771$

1804

1911

$1959 / 1960$

$2253 / 2254$

$2296 / 2297$

$2379 / 2380$

$2456 / 2457$
Close double with LHS 9. Sep. $=2.1^{\prime \prime}$. The pair is also $\mathrm{V}^{*} \mathrm{UV}$ Cet

Kapteyn's star

Proxima. Is also HIC 70890

Sep. $=9^{\prime \prime}$

Close double, Sep. $=0.7^{\prime \prime}$ (ADS 520).

Is also LFT 55, CD-25 225, HIC 2941

IDS 03160-6253, Sep. = 309"

Close

double, Sep. $=2^{\prime \prime}$ (IDS 06549-4409). Is also HIC 33499, Ci 20407

Sep. $=6.4^{\prime \prime}$

Sep. $=16.4^{\prime \prime}$

ADS 9446, Sep. $=22.5^{\prime \prime}$

Sep. $=7^{\prime \prime}$

IDS 17092-2627, Sep. = 5.4"

Is also HIC 84405, GJ 663

Close double IDS 17121-3453AB. Sep. = $1.8^{\prime \prime}$. Is also LFT 1336, HIC 84709, GJ 667 Sep. $=32^{\prime \prime}$

Close

double, Sep. $=2^{\prime \prime}$ (IDS 20372-1918). Is also LFT 1562, GJ 800

Close double, Sep. = $0.1^{\prime \prime}$ (IDS 22160-7244)

Sep. $=51^{\prime \prime}$

IDS 00288-3532, Sep. $=5.2^{\prime \prime}$

Close double, Sep. = $0.1^{\prime \prime} \quad(\mathrm{IDS}$ 00324-3750). Is also HIC 2944, LTT 330

Close double: Rodgers A.W. \& Eggen O.J. 1974

IDS 02008-2833, Sep. $=58^{\prime \prime}$

Close

double, Sep. $=0.5^{\prime \prime}$ (IDS 02243-2026). Is also HIC 11565, LFT 213, GJ 100

IDS 02500-3618, Sep. $=36.7^{\prime \prime}$

Close double (ADS 2402). Is also LFT 261, CD-29 1177, Ci 20 211, HIC 14879, GJ 127

IDS 03276-6317, Sep. $=54^{\prime \prime}$

IDS 05004-5614, Sep. $=79^{\prime \prime}$

IDS 05354-4609, HIC 26501, Sep. $=5^{\prime \prime}$

IDS 05566-3103AB, Sep. $=0.8^{\prime \prime}$.

Is also HIC 28442, GJ 225.2

Close

double, Sep. $=0.6^{\prime \prime}$ (IDS 07146-4649). Is also LFT 521, HIC 35296, GJ 269

IDS 07559-6002, Sep. $=61^{\prime \prime}$

IDS 10188-5939, HIC 50808, Sep. = 9.4"

Sep. $=16^{\prime \prime}$

IDS 11104-1734, Sep. $=18.5^{\prime \prime}$

IDS $11422-6512$, Sep. $=16.3^{\prime \prime}$ 
Close double, Sep. $=1.9^{\prime \prime}$ (IDS 12479-1758). Is also LTT 4924, HIC 62882

$2806 / 2807$ IDS $13432-3512$, Sep. $=11.8^{\prime \prime}$

2815 Possibly close double. Is also LFT 1044, CPD-56 5970, Ci 20815

LHS

3427

3635

3707

$3750 / 3751$

3790

$3921 / 3922$

4013

$5139 \mathrm{a}$
Very large disagreement between proper motions PPM or Luyten and Catalog of proper-motion stars III (Eggen 1987) $(-0.585-0.245)$ or permutation between $\mu \alpha, \mu \delta$

Close double (IDS 20588-7334). Is also HIC 104440, GJ 818.1

Close double (IDS 21395-5808). Is also LFT 1657, CPD-58 7893, HIC 107522

Sep. $=10.3^{\prime \prime}$

Close double, Sep. $=2^{\prime \prime}$ (IDS 22117-5406). Is also LFT 1702, HIC 110109, GJ 853A

IDS 23110-6727, Sep. $=70^{\prime \prime}$

Is also CD-42 16457, LTT 9698, LFT 1823, V* SX Phe (Mv: 6.8/7.5) Close double, Sep. $=1.9^{\prime \prime}(\operatorname{ADS} 6914)$.
Is also BD-22 2345, Ci 20 480, HIC 42430

5299a Is also IDS $15410-3736 \mathrm{~A}, \mathrm{CD}-37$ 10500A, Ci 20 946, GJ 599A, HIC 77358

5299b Is also IDS 15410-3736B, GJ 599B LHS 5299a/5299b, Sep. $=15^{\prime \prime}$

5399 Close double, Sep. = $1.4^{\prime \prime}$ (IDS 23107-4506). Is also HIC 114885, CD-45 14980.

\section{References}

Bessel M.S., 1990, A\&AS 83, 357

Eggen O.J., 1987, AJ 92, 379

Luyten W.J., 1979, University of Minnesota

Rodgers A.W., Eggen O.J., 1974, PASP 86, 742

Rousseau J.M., Périé J.P., Gachard M.T., 1995, Hémisphère Sud Catalogue de Bordeaux, Observatoire de Bordeaux, Vols. 1-5

Rousseau J.M., Périé J.P., Gachard M.T., 1996, A\&AS 116, 301

Rousseau J.M., Périé J.P., 1996, A\&AS 115, 517

Turon C., et al., 1992, The Hipparcos Input Catalogue, ESA SP-1136, Vols. 1-7 